This item was submitted to Loughborough's Research Repository by the author.

Items in Figshare are protected by copyright, with all rights reserved, unless otherwise indicated.

\title{
The study of a bi-stable wake region of a generic squareback vehicle using tomographic PIV
}

PLEASE CITE THE PUBLISHED VERSION

http://dx.doi.org/10.4271/2016-01-1610

\section{PUBLISHER}

(C) SAE International

\section{VERSION}

VoR (Version of Record)

\section{PUBLISHER STATEMENT}

This work is made available according to the conditions of the Creative Commons Attribution-NonCommercialNoDerivatives 4.0 International (CC BY-NC-ND 4.0) licence. Full details of this licence are available at: https://creativecommons.org/licenses/by-nc-nd/4.0/

\section{LICENCE}

CC BY-NC-ND 4.0

\section{REPOSITORY RECORD}

Perry, Anna K., Mathew T. Almond, Martin A. Passmore, and Rob Littlewood. 2016. "The Study of a Bi-stable Wake Region of a Generic Squareback Vehicle Using Tomographic PIV”. figshare. https://hdl.handle.net/2134/21527. 


\title{
The Study of a Bi-Stable Wake Region of a Generic Squareback Vehicle using Tomographic PIV
}

\author{
Anna-Kristina Perry, Mathew Almond, and Martin Passmore \\ Loughborough University \\ Robert Littlewood \\ LaVision
}

\begin{abstract}
This paper demonstrates the use of large scale tomographic PIV to study the wake region of a Windsor model. This forms part of a larger study intending to understand the mechanisms that drive drag force changes when rear end optimizations are applied. For the first time, tomographic PIV has been applied to a large airflow volume $\left(0.125 \mathrm{~m}^{3}, 500 \times 500 \times 500 \mathrm{~mm}\right)$, which is of sufficient size to capture the near wake of a $25 \%$ scale Windsor model in a single measurement. The measurement volume is illuminated using a $200 \mathrm{~mJ}$ double pulsed Nd:Yag laser fitted with a volume optic and seeded with $300 \mu \mathrm{m}$ helium filled soap bubbles generated by a novel high output seeder. Images were captured using four 4M Pixel LaVision cameras. The tomographic results are shown to produce high quality data with the setup used, but further improvements and tests at higher Reynolds number could be conducted if an additional seeding rake was used to increase seeding density. Proper Orthogonal Decomposition (POD) was applied to both the 2D and tomographic data to show the presence of a bi-stable wake structure.
\end{abstract}

CITATION: Perry, A., Almond, M., Passmore, M., and Littlewood, R., "The Study of a Bi-Stable Wake Region of a Generic Squareback Vehicle using Tomographic PIV," SAE Int. J. Passeng. Cars - Mech. Syst. 9(2):2016, doi:10.4271/2016-01-1610.

\section{INTRODUCTION}

As vehicle emissions have moved to the forefront of global legislation the initiative for lighter vehicles and improvements to conventional, hybrid, fuel cell and EV powertrains, has become a primary design objective for manufacturers. However, these advances also call for a more refined aerodynamic package to maintain vehicle stability in cross wind conditions and to reduce the drag to lower the energy consumption and increase range.

The squareback vehicle is of particular interest in the European market due to its popularity for small hatchback vehicles, estates and SUVs as well as commercial vehicles because of the large cabin and load space that they provide. Square-back vehicles are characterized by a large wake volume and associated suction region on the base that contributes up to $30 \%$ of the aerodynamic drag [1]. When a time averaged result is observed the wake is described as toroidal in structure [2] and contained by shear layers emanating from the vehicle sides, roof and floor. However, its highly three dimensional and unsteady nature leaves many questions to be answered regarding the relationship between the wake structures and base pressure. Ahmed [ 3 ] originally showed the presence of upper and lower counter-rotating vortices on the vehicle centerline in a wide ranging paper on wake structures. This result has regularly re-appeared in time average flow structures for square-backs, including in the work of Littlewood \& Passmore [4]. Here a more detailed picture of the near wake along the vehicle centerline was found using planar PIV where the time averaged field was compared to the instantaneous images that were taken to construct it. From this it was shown that while the time averaged structure may be a useful overall description, the dominant vortex cores are not seen to exist as a coherent structure within the wake field instantaneously.

Duell and George [드 found that at the trailing edge of a squareback geometry the boundary layer is shed as vortices over a range of frequencies which are then paired as they are convected downstream, reducing the characteristic frequency of the shear layer and giving some indication of the unsteadiness within the wake. This shedding results in a longitudinal fluctuation at the free stagnation point as these vortices are shed, giving the wake a "pumping" motion. Littlewood and Passmore [4] described the shedding process as a 'Markov chain'.

Volpe, Devinant \& Kourta [6] report pressure measurements and planar PIV measurements in the wake of a $25 \%$ scale model of a squareback Ahmed geometry at Reynolds numbers of $5.11 \times 10^{5}$ and $7.7 \times 10^{5}$. They demonstrated that the wake is a bi-stable structure, switching between sides in the horizontal plane. In this case the complete wake topology was created through the interpolation of multiple planes of two component PIV data that were conditionally averaged in to the "left" and "right" conditions. They also showed that when data is collected for a sufficiently long period the time averaged result returns to the traditional steady state symmetrical 
torus. When the bi-stable wake conditions are compared to the base pressures, the entire wake is in suction but a lower pressure is associated with the larger lobe of the torus structure.

The work of Volpe, Devinant \& Kourta []ㅡ also shows that there is a strong sensitivity to small yaw angles $\left( \pm 0.5^{\circ}\right)$ with any deviation from $0^{\circ}$ resulting in a bias of the bi-stability to the leeward side of the model and the time averaged result no longer seen to be symmetric. With angles of $\pm 1^{\circ}$ there is no longer any hint of bi-stability in the wake structure with a complete shift towards the leeward condition.

The complexity and three dimensional nature of the wake makes it desirable to capture the whole wake structure in a single measurement. This would allow an investigation of the torus structure in both the instantaneous and time averaged fields, whilst also resolving the unsteady nature of the field. Particle Image Velocimetry( PIV) techniques have been around for many years and have increased in capability, with larger fields of view possible and stereoscopic techniques widely reported. However, with the increasing power of computers combined with increased resolution and sensitivity of CCD and CMOS sensors it has become realistic to apply tomographic PIV (3 component volume PIV) techniques to a large volume.

The use of two component, two dimensional PIV is well documented in a variety of situations $[\underline{4}, \underline{7}, \underline{8}, \underline{9}, \underline{10}]$ and traditionally involves a single camera that is normal to the measurement plane, a pulsed laser passing through a plano-concave lens to create a diverging sheet of light focused to a thickness of around $1 \mathrm{~mm}$, neutrally buoyant seeding particles (such as DEHS solution which has been atomized to form $1 \mu \mathrm{m}$ particles) and a computer containing a timing board that allows for the control of the system. This technique is particularly accurate when there is only a small amount of through plane motion so that the majority of the particles captured by the camera in the double frame image pair remain within the light sheet.

Once there is a sizeable amount of out of plane motion, then stereoscopic PIV should be considered. This requires an additional camera in the setup with both cameras focusing on the same field of view with, ideally, an angle of greater than $30^{\circ}$ between them. The cameras are corrected for the schiempflug principle [11] and through the use of a $3 \mathrm{D}$ calibration plate the perspective of the cameras can be used to compute the out of plane velocity component, resulting in a three component, planar (2D) technique. This added component to the velocity however is costly in both processing time and data volume.

Both these two dimensional techniques resolve the velocity in a slice through the wake structure with a thickness determined by the laser sheet. If sufficient planes are acquired the full volume can be reconstructed, however, this is time consuming and even then may not provide the data resolution required and it does not produce a full field instantaneous image. The alternative is to use a volume method that employs multiple cameras and allows each of the 2D camera images to be reconstructed into a three dimensional volume. Such tomographic reconstruction algorithms have been around for many years, perhaps the most relatable modern day example is an MRI machine or a CAT scanner taking multiple two dimensional images through a subject and then reconstructing to form a three dimensional volume.
Tomographic reconstruction techniques have also been used in PIV but typically only for a relatively small volume due to the experimental limitations associated with the camera sensitivity and available light. There are several available algorithms to reconstruct the planar images into a three dimensional volume and these have been analyzed by Elsinga et al. [12]. All such techniques are computationally expensive and should only be considered when a highly three dimensional flow is under investigation, however the technique does give a three component, three dimensional velocity field.

A main limitation to scaling tomographic PIV up to volumes sufficiently large for studying typical automotive problems is the intensity of light reflected from the seeding particles, particularly when the volume is large in all three dimensions. This paper utilizes a new seeding medium, employing $300 \mu \mathrm{m}$ helium filled soap bubbles to capture a large volume $(500 \times 500 \times 500 \mathrm{~mm})$ and apply tomographic techniques to the highly three dimensional squareback wake structure of a Windsor model. The results are compared to planar 2D PIV data.

\section{EXPERIMENTAL METHODOLOGY}

Testing was performed in the Loughborough University Wind Tunnel, full details of which can be found in Johl, Passmore \& Render [13]. The operating velocity in the test section was up to $40 \mathrm{~m} / \mathrm{s}$. The freestream turbulence intensity is approximately $0.2 \%$ and flow uniformity $\pm 0.4 \%$.

\section{Model}

A quarter scale Windsor Model, Figure 1, as used in $[\underline{4}, \underline{7}, \underline{8}, \underline{14}, \underline{15}]$ was used for this work. The model is constructed of a machinable model board and gives a blockage ratio of $4.4 \%$ in the $2.5 \mathrm{~m}^{2}$ working section. The model has large leading edge radiuses $(0.05 \mathrm{~m})$ to prevent local separation and the windshield to roof leading edge has a radius of $0.2 \mathrm{~m}$. The longitudinal and rear edges are all sharp.
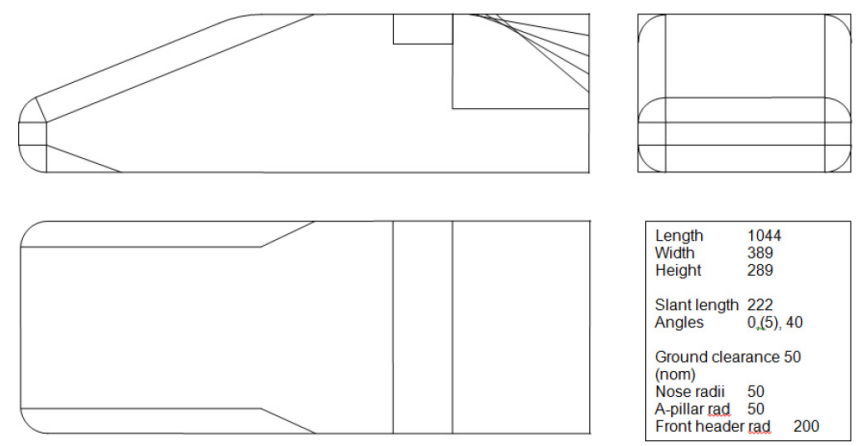

Figure 1. Dimensions of Windsor model

The model was supported by four pins of M8 threaded bar in locations representative of front and rear axles and $10 \mathrm{~mm}$ inboard of the model sides. The ground clearance was kept constant at $50 \mathrm{~mm}$, $(\mathrm{h} / \mathrm{H})=0.17$, which is typical of an MPV ride height. 


\section{Planar PIV Measurements}

Two component PIV measurements using a LaVision system were taken in vertical and horizontal planes in the wake of the model. In the vertical plane, the locations are $\mathrm{y}=0 \mathrm{w} / \mathrm{W}$ (centerline) and $\mathrm{y}=0.26 \mathrm{w} / \mathrm{W}$, seen in yellow in Figure 2. In the horizontal, seen in purple, the planes were located at $\mathrm{z}=-0.67 \mathrm{~h} / \mathrm{H}$ (model centerline) and $\mathrm{z}=-1.11 \mathrm{~h} / \mathrm{H}$.

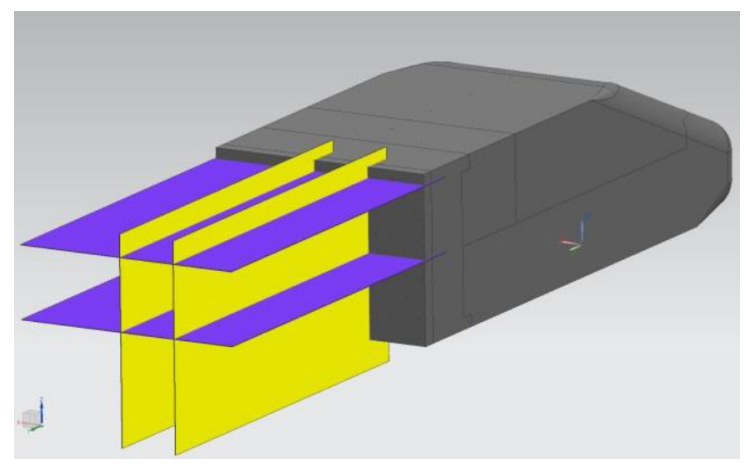

Figure 2. Windsor model showing the locations of 2D PIV planes

For the planar measurements the airflow was seeded using a PIVPart45 aerosol generator, using DEHS (Di-Ethyl-Hexyl-Sebacat) as a seeding fluid. The particles, which are $1 \mu \mathrm{m}$ in size, are introduced in the settling chamber of the wind tunnel using an 8 tier seeding rake. The seeding particles were illuminated by a $200 \mathrm{~mJ} /$ pulse Nd:YAG laser located above the working section. The laser emits a circular beam of $6 \mathrm{~mm}$ in diameter which when passed through a plano-concave cylindrical lens produces a sheet of approximately $1 \mathrm{~mm}$ thickness at the measurement plane. Two Imager ProX four Mega-Pixel cameras, (2048x2048 pixels) were used to gather images. Each camera was fitted with a $50 \mathrm{~mm}$ Nikon lens with an aperture value of $\mathrm{f}-4$. Optical access was provided through the glass working section walls and a glass roof section. The cameras were mounted in a dark room beside the test section to reduce light pollution; in addition $532 \mathrm{~nm}$ band pass filters were fitted to both cameras ensure the highest quality images. A schematic of the experimental set up can be seen in Figure 3 .

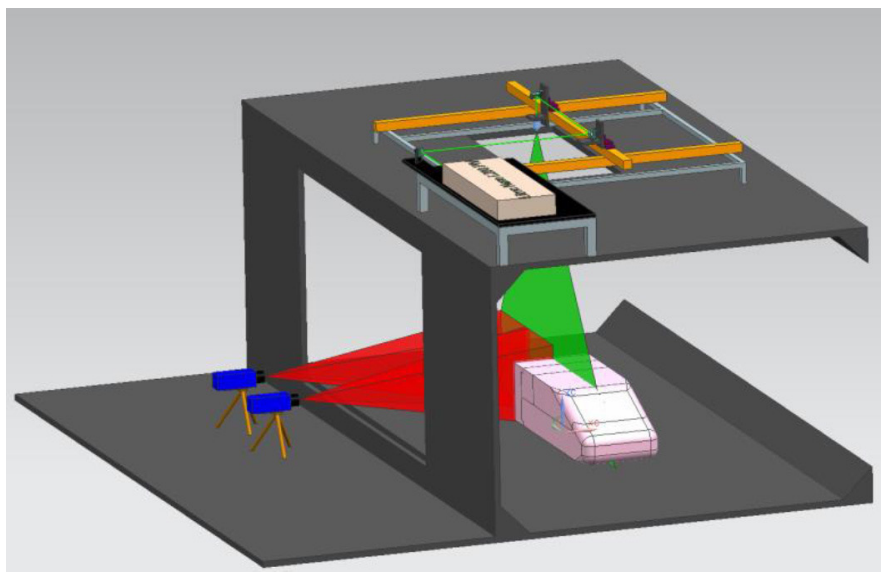

Figure 3. Planar PIV system setup

The laser and camera were controlled by an internal Programmable Timing Unit (synchronizer), which sets the trigger rate at $7.26 \mathrm{~Hz}$. The interframe time $(d t)$ was optimized for each setup position but was typically between $28-30 \mu$ s. Spatial calibration was conducted using a 2D calibration plate that consists of a series of crosses that provide known dimensions in $\mathrm{x}$ and $\mathrm{y}$ for the images to be calibrated against. To verify the PIV system settings were correct, 50 image pairs were taken over different $d t$ values and processed. The processed data was then checked for peak locking and pixel biasing effects by observing the Probability Density Function (PDF) and scatter plots of pixel displacement. One thousand image pairs were taken for each test configuration and were processed using LaVision's DaVis 8.0 software on a graphical processing unit (GPU) using direct correlation.

Additional tests taking 2000 image pairs showed no significant improvement in the statistical uncertainty of the PIV data [16].

Processing was performed using a multi-pass processing technique, starting at a window size of $128 \times 128$ pixels and reducing to a window size of $24 \times 24$ pixels. Seven passes were done in total. The overlap between the interrogation windows was kept constant at $50 \%$. To reduce the chance of spurious vectors, the parts of the raw image where the model, tunnel floor or any other obstruction could be seen were masked. This left only the area of interest in the processed vector fields.

\section{Tomographic PIV Measurements}

A tomographic volume measuring $500 \times 500 \times 500 \mathrm{~mm}$ was used in the near wake of the vehicle model. The purpose was to encompass the whole vehicle wake, along with part of the freestream above and the flow exiting from underneath the model. Figure 4 shows the location of the tomographic measurement volume in relation to the Windsor model.

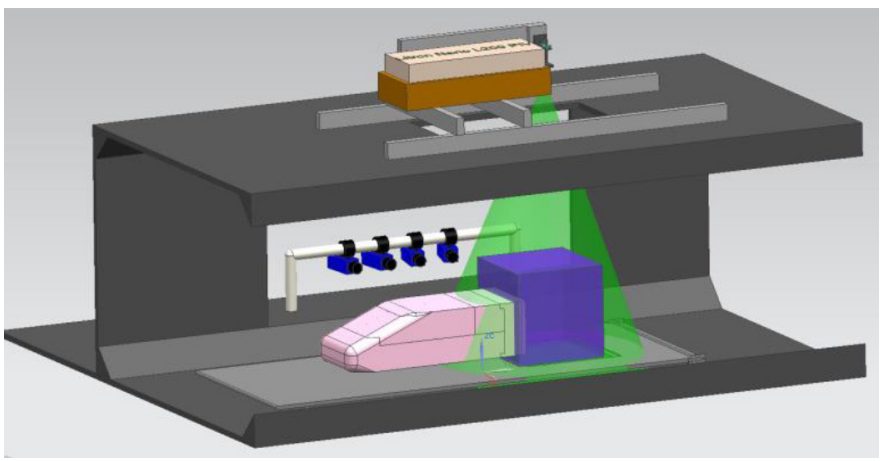

Figure 4. Tomographic PIV system setup with purple box representing the measurement volume

Two extra cameras were used for the Tomographic PIV setup, each set to the same four Mega-pixel resolution as used for the planar PIV. The four cameras were mounted on a frame in a horizontal arrangement (see Figure 4).

Three or more cameras are recommended for full 3D PIV because of the tomographic reconstruction of a volume of particles from multiple $2 \mathrm{D}$ camera images. In the work reported here the tomographic reconstruction of the particle images used the LaVision 'FastMART' algorithm which employs an approach similar to that described by Atkinson \& Julio [17]. While the process works with only three cameras, increasing to four (or more) increases the accuracy of the 
direct correlation result because it reduces the number of occurrences of single particles being identified as multiple particles (ghost particles).

The system is calibrated using a $300 \mathrm{~mm} \times 300 \mathrm{~mm} 3 \mathrm{D}$ calibration plate placed at the front, middle and rear of the volume of interest relative to the camera positions. During both calibration and the experiments the cameras used an aperture of $f-8$ to ensure suitable focus is maintained throughout the full depth of the volume. Images of the calibration plate are used in a camera pinhole mapping algorithm [11] that maps the $3 \mathrm{D}$ space visible to the cameras. The quality of the calibration is checked by de-warping the raw camera images onto a flat plane corresponding to the physical measured location of the calibration plate faces. A simulated regular grid is plotted on the same location with a grid spacing that corresponds to the spacing of the machined dots on the calibration plate. The alignment of the dots in the de-warped images of the calibration plate onto a regular grid is analyzed and the RMS error for each calibrated location and image is reported.

In $2 \mathrm{D}$ or Stereo PIV an RMS error below 0.5pixels is acceptable; however in Tomographic PIV an error below 0.1pixels is required to yield reliable results. Often this is not achievable simply using images of a calibration target, so an additional volume self-calibration procedure [18] is used to refine the calibration using the actual experimental data. This process uses $2 \mathrm{D}$ particle images from each camera taken at the same point in time, i.e. such that there should be no movement of the particles. If the calibration process was perfect, then once the particles positions are triangulated there should be no difference in their physical location as seen by all the cameras. The error for each particle at various locations in the calibrated volume is calculated for each camera and plotted as a vector. The vectors are then used to re-map the calibration and bring the overall RMS errors down below 0.1 pixels. In the work reported here the final RMS errors were 0.004 .

Seeding for the tomographic measurements was generated using a novel high output helium filled soap bubble (HFSB) seeder. This used compressed helium and air to create bubbles of $300 \mu \mathrm{m}$ in size, introduced into the contraction of the wind tunnel using an airfoil shaped seeding rake (pictured in Figure 5). The increased particle size was required to improve the light scattering in such a large measurement volume. The intensity of the scattered light squares with particle size, so a 300 times increase in particle size meant the particle images in the tomographic tests were 90,000 times brighter than the standard seeder producing $1 \mu \mathrm{m}$ particles.

To ensure that seeding particles are entrained into the wake from the flow exiting from under the model a raised ground was installed into the working section $50 \mathrm{~mm}$ above the standard floor. The ground board is a $20 \mathrm{~mm}$ thick flat plate supported by $12 \times 50 \mathrm{~mm}$ steel spacers, mounted through the wind tunnel floor. The boundary layer thickness on the ground plane at a location corresponding to the leading edge of the model was calculated to be $19 \mathrm{~mm}\left(\delta^{99}\right)$.

To illuminate the particles in the measurement volume the beam from the $200 \mathrm{~mJ} /$ pulse Nd:YAG laser was expanded using a volume optic to create a 3D conical output. Expanding the laser in two dimensions reduced the power density in the measurement volume, so a mirror was placed under a Perspex section of the ground board to recycle part of the laser power back into the measurement volume.

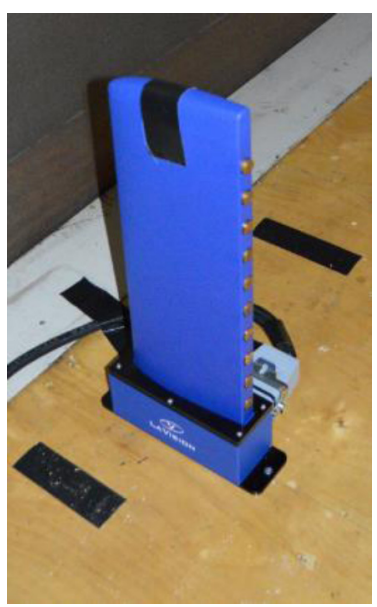

Figure 5. Helium Filled Soap Bubble Seeder mounted in contraction of Wind Tunnel

The current tests were conducted in an open return wind tunnel which means that any seeding produced is not recycled as may be the case in a closed return tunnel. Further, the use of helium bubbles would mean that the even in a closed return tunnel the bubbles would not survive the journey through cleaning meshes or heat exchangers. The main challenge in the design of the seeder was to produce neutrally buoyant bubbles at high enough production rates. The seeder used here produced 300,000 bubbles per second per nozzle from a rake consisting of 10 nozzles. This production rate is orders of magnitude higher than any other devices of this type. However, during the experiments reported here the test speed was limited to $30 \mathrm{~m} / \mathrm{s}$ to ensure sufficient seeding density. At $30 \mathrm{~m} / \mathrm{s}$ the current seeding rate should produce in the order of 50,000 particles in each $500 \mathrm{~mm} x$ $500 \mathrm{~mm} 2 \mathrm{D}$ image. On average, in the $2 \mathrm{D}$ views acquired in this work approximately 56,000 bubbles were measured. For larger volumes or higher tests speeds additional seeding rakes can be added.

In practice 50,000 particles per image is somewhat less than would normally be preferred for tomographic PIV experiments. To ensure good quality vector fields the final grid resolution was reduced using a final PIV window size of 112 x 112 x 112 pixels with $75 \%$ overlap; this results in a vector grid resolution of $10 \times 10 \times 10 \mathrm{~mm}$. Higher resolution could be achieved using multiple seeding rakes but the data yield over the $500 \times 500 \times 500 \mathrm{~mm}$ volume is still considerable.

\section{Proper Orthogonal Decomposition (POD)}

Proper Orthogonal Decomposition (POD) is a powerful data analysis method in turbulence research [19], and is particularly suited to large data volumes which represent non-linear phenomena such as the highly three-dimensional vehicle wakes investigated here. POD takes the input data and decomposes the fluctuating component by creating a basis of spatial functions, $(\phi(x))$, which are optimized solutions to the Fredholm equation and uncorrelated temporal coefficients, $a_{n}(t)$, shown in Equation 1. $\mathrm{N}$ is equal to the number of samples taken and hence the number of modes in the decomposed field. The eigenvalue 
associated with each mode then represents the turbulent kinetic energy contained within that mode, thereby allowing the most dominant flow structures in the low order POD modes to be investigated free from the high order turbulence. The application of POD is covered extensively by Tropea et al.[20].

$$
u(x, t) \cong \sum_{n=1}^{N} a_{n}(t) \phi_{n}(x)
$$

\section{PLANAR PIV RESULTS}

For the squareback Windsor Model, the planar PIV results were collected at a tunnel speed of $40 \mathrm{~m} / \mathrm{s}$ and a frequency of $7.26 \mathrm{~Hz}$, equating to 134 seconds of data. As this is low speed PIV the vector fields are uncorrelated. However, the constant sampling frequency does allow for time dependent decompositions to be performed.

The 2D time averaged results are presented in Figure 6 , in all cases the velocity has been normalized using $\mathrm{V}_{\text {Tunnel }}$. In Figure 6a, the vertical centerline wake data is shown. The wake is $0.43 \mathrm{l} / \mathrm{L}$ long at the free stagnation point and the upper vortex core has a slight dominance over the lower. In the horizontal mid-plane, Figure 6b, the vortex cores are biased towards the left hand side of the model; this asymmetric time averaged result is a consequence of a bistability of the wake structure. Such bi-stability has been discussed in some detail by Volpe et al []] and Pavia, Passmore \& Gaylard [1] . Because the bi-stability occurs irregularly with a long time period, the total sample of 134 seconds is insufficient to produce a symmetric flow field. Figure 7 shows a POD reconstruction from the first 50 modes, which contains approximately $50 \%$ of the flow field's turbulent kinetic energy. There are two distinctive conditions of the wake - a left (igire 7a $)$ ) and a right mode ( $\underline{\text { Figure } 7 \mathrm{~b}})$ ). The amount of time spent in each state is known to be random [6] , however the plot of the temporal coefficient of the first POD mode (containing $15 \%$ of the turbulent kinetic energy) shows the "switching" of the modes, Figure 7c).

POD analysis of the vertical centerline plane (plots not shown) does not show any of this bi-stable mechanism in the temporal coefficient or first five Eigen modes; instead a pumping mode of the wake is seen, previously demonstrated by Duell \& George [ $\underline{5}]$, in the eigenvalue field of the first mode.

Considering the time averaged plots further, the off center vertical plane, Figure $6 c$, shows part of the wake side structure. There is a source point highlighted by the red circle as flow travels from the bulk structures inside the wake and out towards the shear layers emanating from the sides of the model and similarly there are two sink points, shown in yellow, as flow travels from near the model sides towards the central wake region. POD analysis on this wake plane also highlights two modes, essentially an "on" mode and an "off" mode. In the "on" mode there is a structure indicating that the wake is biased towards the right hand side of the model, while in the "off" mode, significantly less flow is seen in this region indicating that the wake has shifted towards the left hand side of the model and only the edge of the shear layer is captured. Again this shift is easily recognized in the temporal coefficient of the first mode.

Finally in the upper horizontal plane, Figure 6d, there is a single source point. This might be assumed to be centrally located within the wake structure but it is biased to the right hand side as a consequence of the bi-stability that is present. POD analysis of this plane confirms the presence of two modes, but to be visible in this and the off center vertical plane suggests the whole wake must be undergoing a twisting motion. If this is the case at the upper trailing edge of the model, it will also be seen at the lower trailing edge of the model, however due to restricted optical access this was not measured.

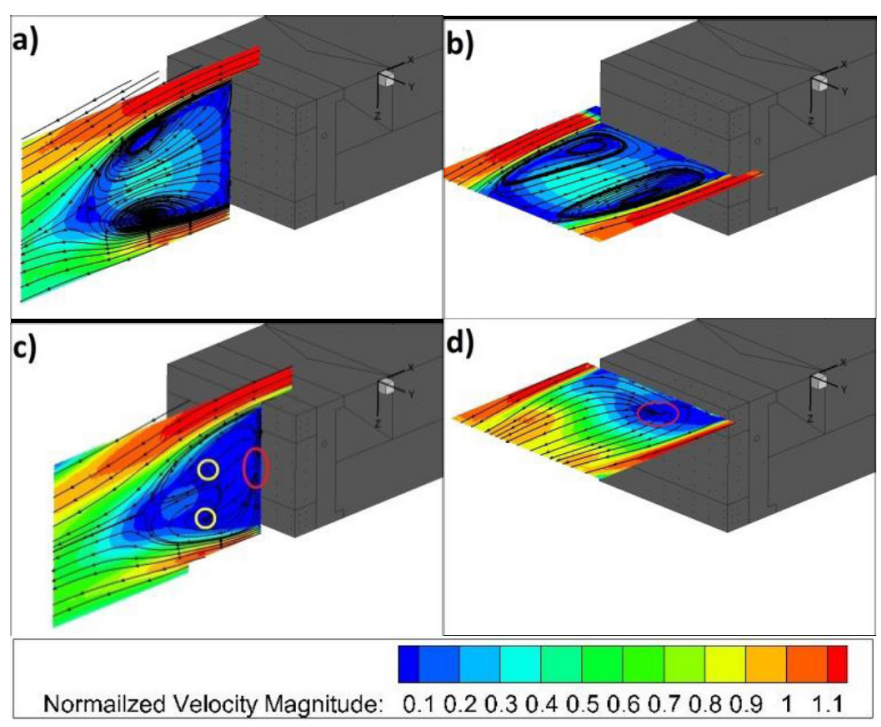

Figure 6. Two Component Planar PIV Time Averaged Results: a) Vertical Centerline, b) Horizontal Centerline, c) Vertical Off Centre ( $\mathrm{y}=100 \mathrm{~mm}), \mathrm{d})$ Upper Horizontal $(\mathrm{z}=-320 \mathrm{~mm})$

Overall these four planes give a clear description of the wake and show the presence of bi-stability that is mainly formed by a shift between the left and right hand sides of the model, seen primarily in the horizontal data plane, with the central vertical portion of wake remaining stable. However, the upper horizontal plane presents the idea of a wake twisting motion rather than a simple left to right switch. This has also been captured by Volpe et al [] $]$ who reconstructed the wake region in its two modes by completing five vertical data planes and five horizontal planes using 2D two component PIV, conditionally averaging and assuming that over the 900s data collection time, sufficient "switches" had occurred for the conditional modes to be statistically accurate. The modes in each plane were then pieced together and an interpolation in the wake volume completed to give an iso-surface of the wake structure. The insight provided by the reconstruction of multiple planes of data is clearly valuable and makes the case for a single volumetric PIV measurement that captures the complete wake. 

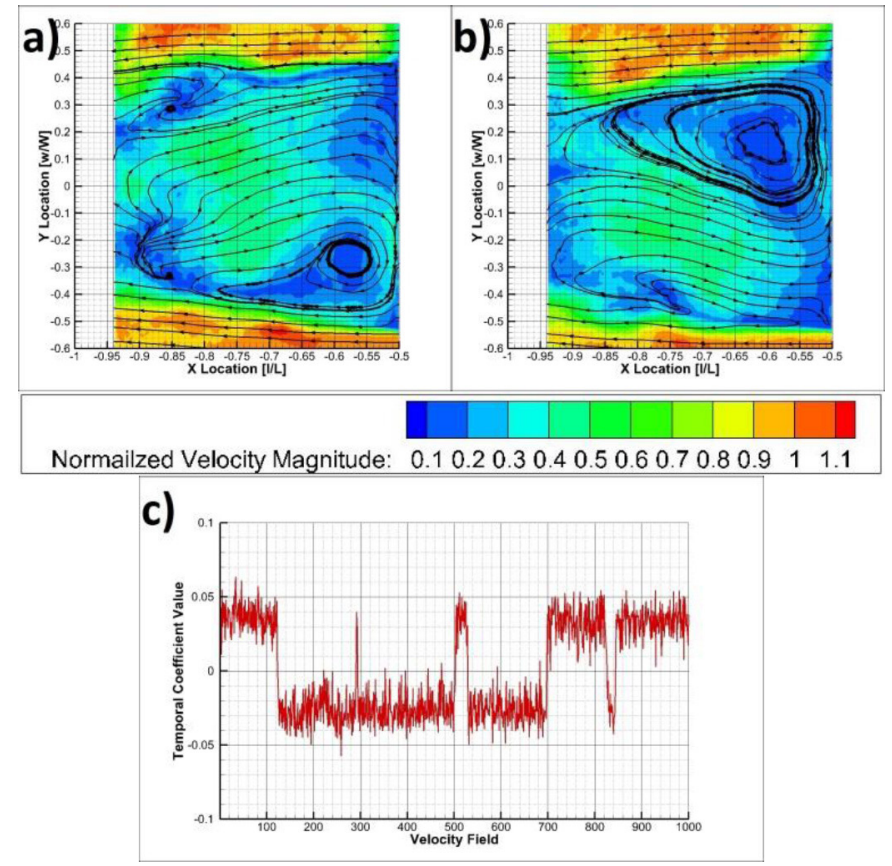

Figure 7. POD analysis on the Horizontal Centerline plane showing two reconstructed fields using 50modes, demonstrating the "left" (a) and "right" (b) bias of the wake structure; the temporal coefficient for the first mode demonstrating switching between conditions is shown in (c).

\section{TOMOGRAPHIC PIV RESULTS}

The tomographic results presented here are the result of the first ever wind tunnel measurements of a large $(500 \times 500 \times 500 \mathrm{~mm})$ PIV volume and as such present only the first steps in developing the use of this method in automotive aerodynamics. The interesting wake structure reported in the 2D results section is clearly an ideal candidate for these first explorations of the technique. Due to the size of the data files that are collected for each instantaneous image, only 400 image pairs were collected rather than the usual 1000. Because of the smaller data set the collection frequency was reduced to $1 \mathrm{~Hz}$, to extend the total sample time in an attempt to capture the bi-stability seen in the $2 \mathrm{D}$ results. The data was also collected at $10 \mathrm{~m} / \mathrm{s}$ for 50 images to compare results with differing seeding densities and provide a level of confidence in the higher speed results.

For this first experiment the requirement to use a separate ground plane removes the possibility to yaw the model during the experiment as the support locations are fixed. This prevented the usual setup procedure whereby the side-force is minimized by imposing a small yaw angle to ensure that the model is completely aligned with the flow in the tunnel.

Figure 8 , shows a sample raw image pair collected by one of the four cameras at $10 \mathrm{~m} / \mathrm{s}$ and $30 \mathrm{~m} / \mathrm{s}$. The reduction in seeding density is apparent, and would have ideally been resolved by including further bubble generators in the tunnel contraction, however due to the limited availability of the prototype parts this was not possible for this particular experiment. Instead this is overcome by increasing the final window size during the direct correlation processing, whilst including a high percentage of overlap between the windows to increase the resolution as much as possible. The inter-frame time was then set to match this large window size such that the particles shifted by approximately $1 / 4$ of an interrogation window.

In Figure 9 , results are shown for the $10 \mathrm{~m} / \mathrm{s}$ and $30 \mathrm{~m} / \mathrm{s}$ tomographic cases and the 2D planar measurements at $40 \mathrm{~m} / \mathrm{s}$ along the vertical and horizontal centerline. The velocity has been normalized using $\mathrm{V}_{\text {Tunnel }}$, and the results can be seen to have good correlation. Each tomographic data set has been produced using the same processing parameters, and although some flow differences are expected due to the changing Reynolds effects, the aim here is to understand the quality of the data with reduced seeding density. The similarities between the two sets of tomographic results, Figure 9a-d, indicates that while the grid resolution is relatively low with high overlap in the processing stage, there appear to be no issues resolving the internal wake flow structures with the lower seeding densities experienced with the $30 \mathrm{~m} / \mathrm{s}$ tomographic data.

The Windsor model in this form is reasonably insensitive to Reynolds Number above approximately $2.5 \times 10^{6}$ and therefore the results shown at $30 \mathrm{~m} / \mathrm{s}\left(\operatorname{Re}=2.6 \times 10^{6}\right)$ and $40 \mathrm{~m} / \mathrm{s}\left(\operatorname{Re}=3.4 \times 10^{6}\right)$ are expected to show similar wake topology; this is evident in Figure 9 even though the resolution of the velocity field is significantly lower for the tomographic data. The vertical centerline plane for the $30 \mathrm{~m} / \mathrm{s}$ data, Figure 9c, shows a large upper recirculation bubble with a smaller lower recirculation region. However, at the base of the model (located at $1 / \mathrm{L}=-0.5$ ) the flow is impinging at a normal angle at approximately $-\mathrm{h} / \mathrm{H}=0.35$, indicating there is a preference to a larger upper vortex structure. However the lower vortex core should be significantly larger than what is seen here. This corresponds with that seen in Figure 9a at $10 \mathrm{~m} / \mathrm{s}$ and in Figure $9 \mathrm{e}$ at $40 \mathrm{~m} / \mathrm{s}$ where there is a dominance of the upper vortex structure. The planar data on this vertical centerline plane also shows the wake closure occurring just downstream of the measurement volume of the tomographic data. In the central wake region there is good correspondence between the topology of the normalized velocity magnitude contours in the planar PIV and the $30 \mathrm{~m} / \mathrm{s}$ tomographic PIV, giving a qualitative assurance to the data.

In the horizontal plane there is good correlation on the normalized velocity magnitude contours between the $30 \mathrm{~m} / \mathrm{s}$ tomographic and $40 \mathrm{~m} / \mathrm{s} 2 \mathrm{D}$ data, with the main difference being the capture of the freestream flow outside of the wake structure. This data is on the edge of the tomographic volume and can be expected to have relatively high errors with the seeding density that was achieved. However, the wake structures are well resolved. At $10 \mathrm{~m} / \mathrm{s}$ and $30 \mathrm{~m} / \mathrm{s}$ the tomographic data shows a clear asymmetry to the recirculation compared to the 2D data; this is unexpected given the long sample time chosen for the tomographic set up and suggests that the model may not be precisely aligned to the wind, impacting the ability to identify the bi-stability. The largest difference between the planar and tomographic data occurs close to the base (up to $-0.551 / \mathrm{L}$ ) showing increased errors in this region. This may be a consequence of the seeding, illumination or the relatively large interrogation regions used in the processing but is an area worthy of further experiment. 

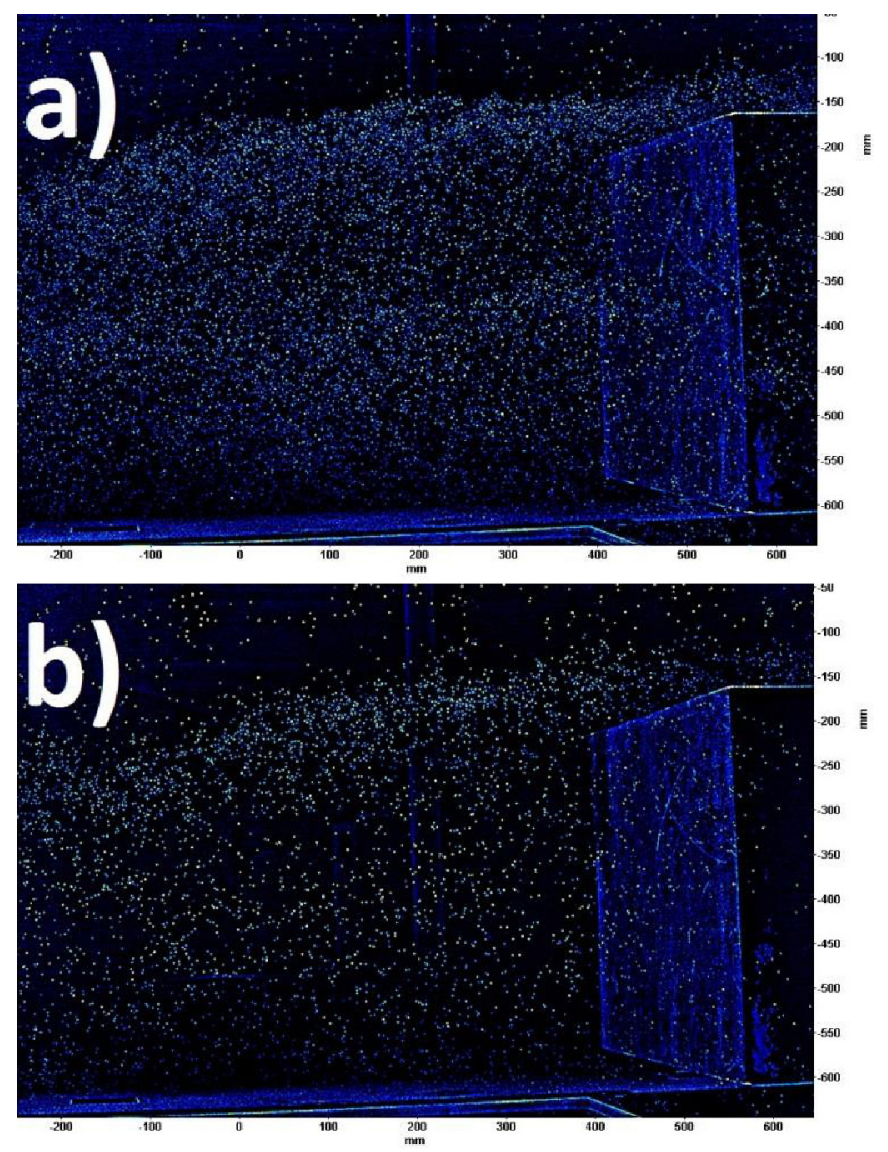

Figure 8. Sample raw data images after initial pre-processing to remove background light had been completed at a) $10 \mathrm{~m} / \mathrm{s}$ and b) $30 \mathrm{~m} / \mathrm{s}$.

A more detailed analysis of the errors was performed by calculating the uncertainty at the $95 \%$ confidence interval for velocity magnitude of the time averaged mean vector field. The uncertainty is the estimate of error in a measurement and is required because the true value of velocity at a given point is unknown. The uncertainty at a particular confidence level is given by Equation 2, where $\varepsilon_{u}$ is the uncertainty value, $t^{*}$ is the distribution factor associated with the desired confidence level for a normal distribution, $\sigma$ is the standard deviation of instantaneous results and $\mathrm{N}$ is the number of samples. In this case $t^{*}=1.960$ for a $95 \%$ confidence level. In regions of high turbulence the uncertainty value will increase, therefore a sufficient number of images need to be taken in order to have confidence in the time averaged result.

$$
\varepsilon_{\mu}=\frac{t^{*} \sigma}{\sqrt{N}}
$$

In Figure 12 (shown in Appendix) the results are shown as contour plots of uncertainty in the vertical centerline of all experimental set ups and for data sets of 50, 400 and 1000 image pairs. The $10 \mathrm{~m} / \mathrm{s}$ tomographic data is included for completeness and to assess the effect of the seeding density. The uncertainty has been normalized using the respective value of $\mathrm{V}_{\text {Tunnel }}$ such that the results can be more fairly compared.
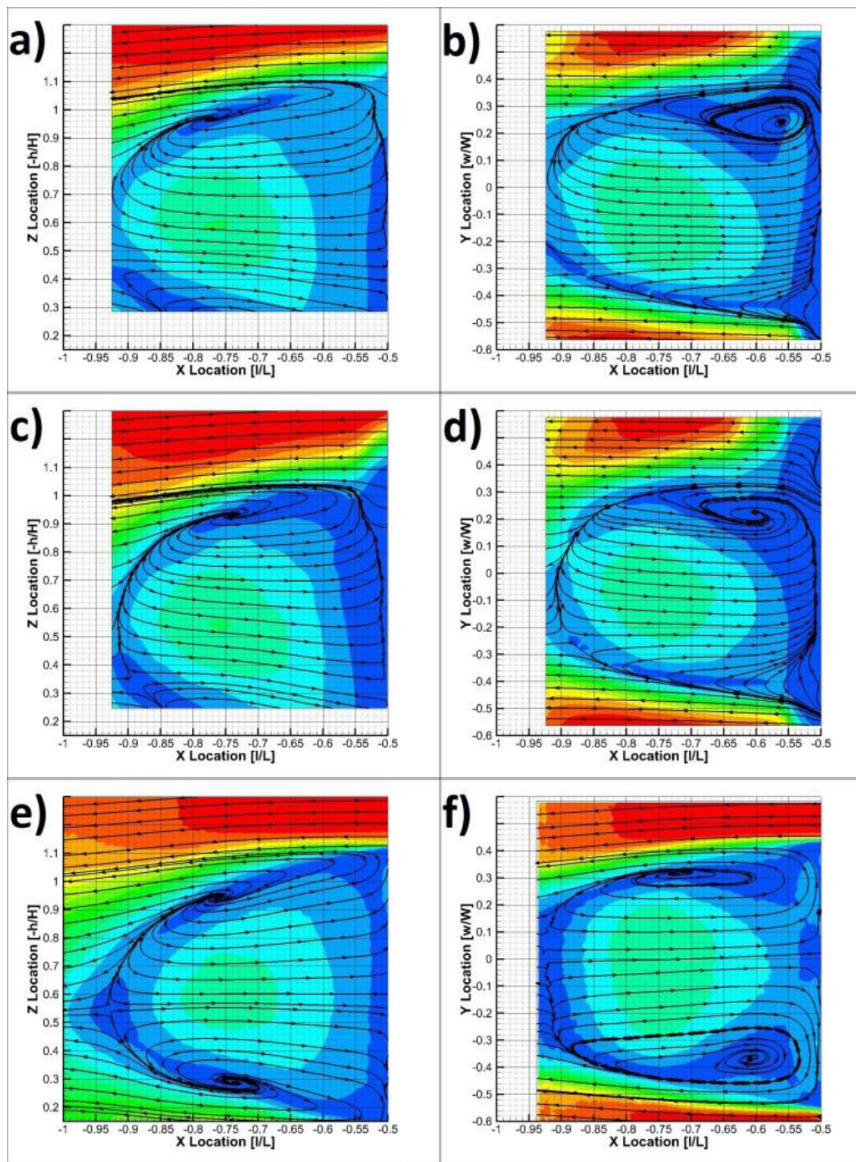

Normailzed Velocity Magnitude: 0.10 .20 .30 .40 .50 .60 .70 .80 .911 .1

Figure 9. Slices through Tomographic Volume obtained at $10 \mathrm{~m} / \mathrm{s}$ and $30 \mathrm{~m} / \mathrm{s}$ compared to 2D data at matching locations: a) $\mathrm{y}=0 \mathrm{~mm} 10 \mathrm{~m} / \mathrm{s}$ Tomographic data; b) $z=194.5 \mathrm{~mm} \mathrm{10m} / \mathrm{s}$ Tomographic Data; c) $\mathrm{y}=0 \mathrm{~mm} 30 \mathrm{~m} / \mathrm{s}$ Tomographic data; d) $z=194.5 \mathrm{~mm} 30 \mathrm{~m} / \mathrm{s}$ Tomographic Data; e) y=0 $\mathrm{mm} 40 \mathrm{~m} / \mathrm{s}$ Planar data; f) $\mathrm{z}=194.5 \mathrm{~mm} 40 \mathrm{~m} / \mathrm{s}$ Planar Data;

Comparing the results presented for a 50 image average it is seen that when increasing the tunnel speed from $10 \mathrm{~m} / \mathrm{s}$ to $30 \mathrm{~m} / \mathrm{s}$ the relative uncertainty in the tomographic data is increased from around \pm 0.04 (4\%) in the main wake region to between $\pm 0.06-0.1$ (6-10\%). For the same sample size this indicates a proportionally larger fluctuating velocity component and is likely a consequence of higher turbulence intensity at the higher Reynolds number. Increasing the number of images used to 400 , it is seen that the uncertainty in the wake region is reduced to $\pm 0.01-0.02(1-2 \%)$.

Considering the planar PIV data, it is seen that these show high levels of uncertainty when only 50 images are considered, particularly within the upper and lower shear layers. However, as sample size is increased to 400 images there is a similar reduction as seen for the tomographic $30 \mathrm{~m} / \mathrm{s}$ case, although with slightly higher uncertainty values seen in the $2 \mathrm{D}$ data $( \pm 0.02-0.03)$. One would normally expect the tomographic data to have the higher uncertainty, because of the additional reconstruction step, but here the $2 \mathrm{D}$ data gives the higher uncertainty because it is analyzed using a much smaller interrogation cell size and therefore captures the smaller turbulent scales. Finally, 
considering the full 2D data set of 1000 images the least uncertainty is seen overall as expected, with a particularly accurate result seen in the near base region, indicating that even if there is through plane motion there are a sufficient number of images to accurately resolve the mean field.

Overall this analysis demonstrates that the data recorded at $30 \mathrm{~m} / \mathrm{s}$ using the tomographic technique is statistically reliable when a mean field of 400 images is considered; this in turn validates the approach of using a large final window size and overlap during processing to reduce the influence of the sparse seeding on the direct correlation.

Figure 10a and Figure 10b show the complete time averaged wake obtained at $30 \mathrm{~m} / \mathrm{s}$ in the form of iso-surfaces combined with $2 \mathrm{D}$ planes on the vertical and horizontal centrelines. The field of view did not allow for the lower wake region to be captured because of reflections from the mirror under the raised ground plane, or for the wake closure to be seen. This was a limitation of this particular set up rather than the experimental technique; future experimentation would need to consider the compromise between a high light intensity over a large volume versus near floor data capture. An alternative approach to using a mirror to increase intensity may be required.

The vector fields in Figure 10a and Figure 10b are the same as those presented in Figure 9c \& Figure 9d and show a dominance of the upper vortex structure in the vertical plane, while the horizontal plane is biased towards the left hand side of the model. The iso-surface, showing $|\bar{V}|=0.99$, gives the outer bounds of the shear layer around three sides of the model. The gap between the side shear layer and the model base is likely due to lack of seeding and therefore data in this region. The overall appearance of the shear layer shows the model to have a largely symmetric wake structure, however the second iso-surface at $|\bar{V}|=0.3$ shown in Figure 10c, shows an off center region of flow representing the inner surface of the wake torus that is not symmetrical. The asymmetry, suggesting the two sides of the torus are not equal in the average, is unexpected for the long sample period so either occurs because of very long time scale bistability or because the model is not sufficiently well aligned with the flow.

Plotting streamlines with seed points at the upstream face of the wake volume, and then integrating through the volume the torus structure of the wake can be clearly seen, Figure 10d and $\underline{10 \mathrm{e}}$. Due to the limitations of the measurement volume, the lower vortex is only partially resolved but tracing the streamlines through the volume, the overall highly three dimensional nature of the wake is very evident. Furthermore if the flow over the top of the model is considered, there is a distinct twist to the structure.

By considering the instantaneous fields, an indication of the unsteadiness of the internal wake structure can be gained. Figure 11 shows four sample images from the data set, demonstrating different flow states. In Figure 11a the central wall of the torus structure is biased towards the left of the wake as seen in the time averaged field. This condition is most prevalent throughout the data set (approximately 95\%). Figure 11b shows this inner wall of the torus to the right hand side of the wake structure. This is characteristic of bi-stable behavior with the wake switching between these two conditions but is only seen in around $2 \%$ of all the images.
However, two further conditions are seen in the dataset; Figure 11c shows a wake condition where the inner wall of the wake torus is located in the center of the wake region as would be expected for a symmetrical flow and in Figure 11d there is a condition where the internal flow structure shows no coherent structure from which an iso-surface of velocity magnitude can be interpolated; this is the least common mode.
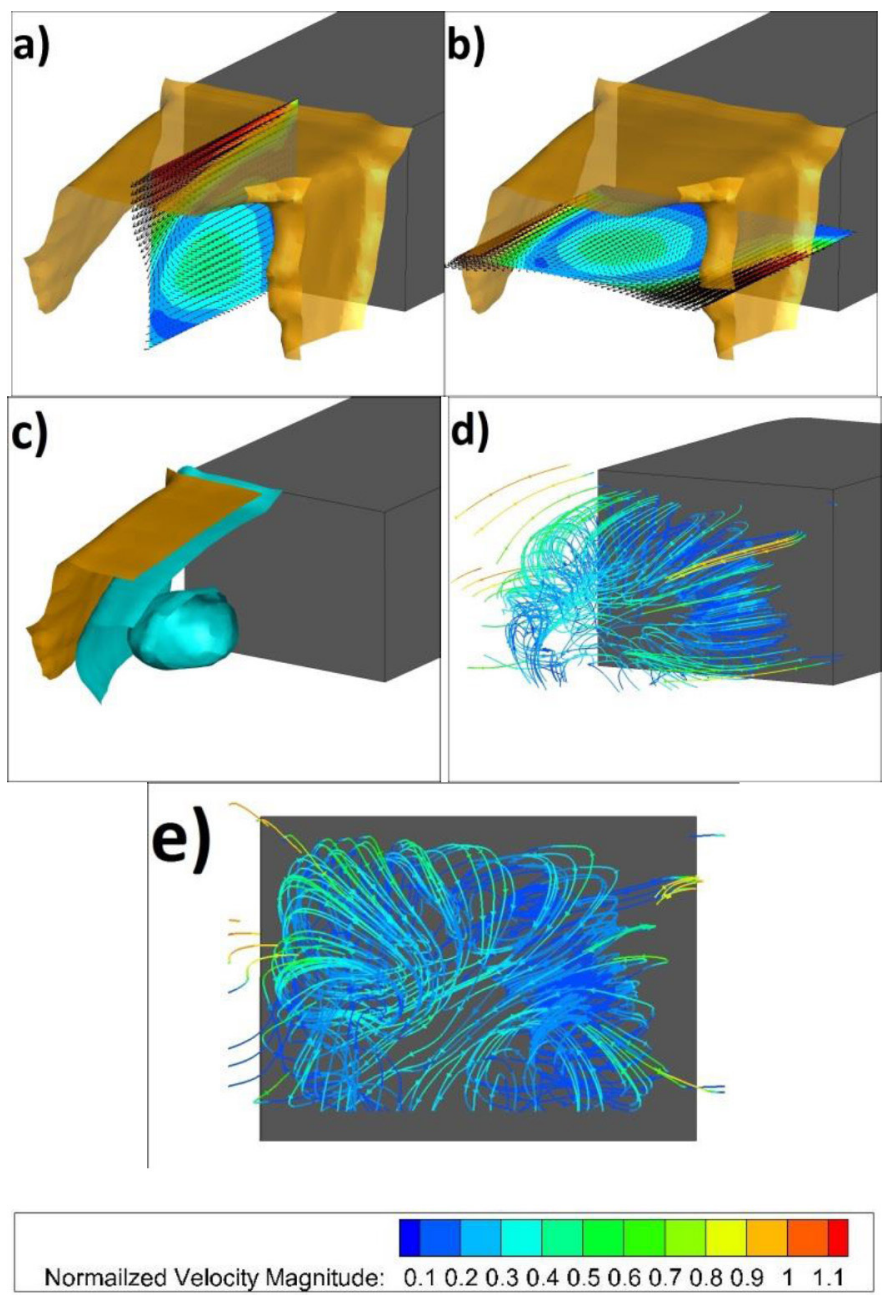

Figure 10a). $|\bar{V}|=0.99$ Iso-Surface with vertical slice at $\mathrm{y}=0 \mathrm{~mm} . \mathrm{b}$ )

$|\bar{V}|=0.99$ Iso-Surface with horizontal slice at $\mathrm{z}=-194.5 \mathrm{~mm}$. c)

$|\bar{V}|=0.99$ and $V \mid=0.3$ Iso-Surface. d) Streamlines colored by normalized velocity magnitude. e) Streamlines colored by normalized velocity magnitude, base view.

When POD analysis is performed over the entire wake volume only $6.5 \%$ of the overall kinetic energy is captured in the first mode and the temporal coefficient shows no bi-stable switching as seen in the 2D PIV data. Highly turbulent flows characteristically have a more even energy distribution throughout the POD modes, while bistability is more commonly seen where there is a large difference between the energy of the first and subsequent modes. To compare directly with the planar results, a single plane of data was extracted at the horizontal centerline (matching the location in the 2D PIV) and then POD analysis was conducted for this plane. The first mode now contained $12 \%$ of the overall kinetic energy (compared with $15 \%$ in 2D) and when the reconstructed fields were individually reviewed it was possible to see that there is a dominance of the left hand mode 
but there is an intermittent "switch" to the central condition, shown instantaneously in Figure 11c. This strongly suggests that there is bistability and if the model was more closely aligned with the flow the two distinct left/right modes seen in Figure 7 would become clear.
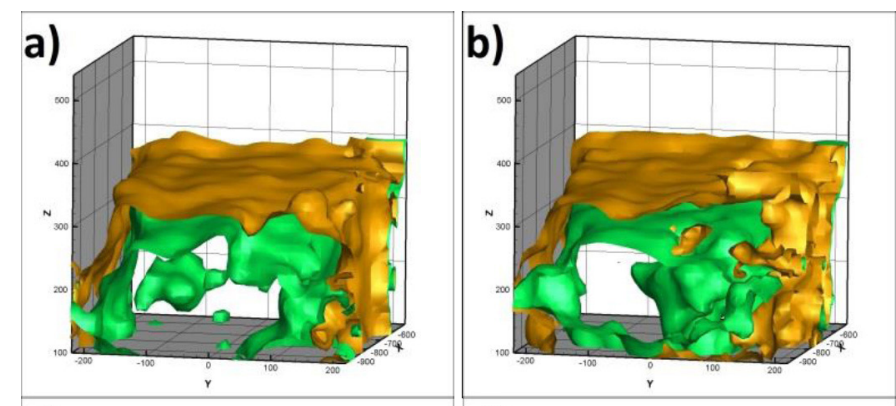

c)
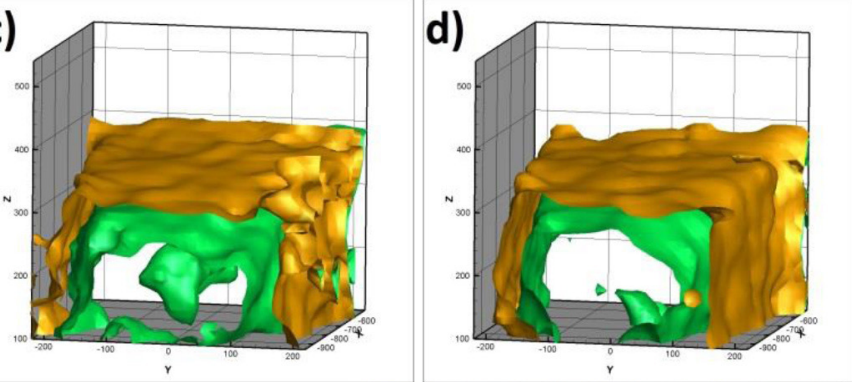

Normailzed Velocity Magnitude:

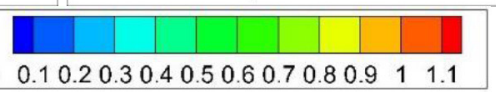

Figure 11. Iso-surfaces presented at $|\bar{V}|=0.99$ and $|\bar{V}|=0.45$ showing 4 instantaneous snapshots.

\section{SUMMARY/CONCLUSIONS}

The work reported shows the results of the first large depth tomographic PIV experiment to be conducted for an automotive geometry. It demonstrates that it is possible to capture a complete 3D-three component wake structure of a quarter scale vehicle model using tomographic PIV at a representative Reynolds Number.

The images obtained are shown to produce high quality results with confidence in the mean velocity fields comparable to the planar 2D test. However, the tomographic data is analyzed with a larger final interrogation cell size introducing additional spatial averaging that limits the number of turbulence scales that are resolved. With more seeding particles it is expected that the tomographic data will produce slightly higher uncertainty than 2D for a given sample size and interrogation cell size because of the additional reconstruction step in the processing.

The application of the experimental technique to the large scale problem described requires further refinement to allow tests at higher Reynolds numbers. This includes the addition of further bubble seeding rakes and alternative methods for increasing the level of illumination in the measurement volume.

The 2D planar results illustrated the wake bi-stability seen by a number of authors. This was not seen directly in the tomographic results but it has been shown that this is likely due to a small misalignment of the model in the wind tunnel. POD analysis of the tomographic data suggests that the bi-stability is present but that it is strongly biased to one side because of the small yaw angle.

For this particular experiment, future work should be completed with the ability to yaw the model so that closer alignment with the onset flow can be achieved and bi-stability recorded more clearly.

The instantaneous tomographic velocity fields show the true unsteady nature of the wake demonstrating the bulk motion within the region encapsulated by the shear layers.

\section{REFERENCES}

1. Irving Brown, Y., Windsor, S., and Gaylard, A., "The Effect of Base Bleed and Rear Cavities on the Drag of an SUV," SAE Technical Paper 2010-01-0512, 2010, doi:10.4271/2010-01-0512.

2. Roumeas, M., Gillieron, P., \& Kourta, A. "Analysis and control of the near-wake flow over a square-back geometry", Computers and Fluids, 38:60-70, 2009

3. Ahmed, S. "An experimental study of the wake structures of typical automobile shapes", Journal of Wind Engineering and Industrial Aerodynamics, 9: 49-62, 1981.

4. Littlewood, R. and Passmore, M., "The Optimization of Roof Trailing Edge Geometry of a Simple Square-Back.," SAE Technical Paper 2010-

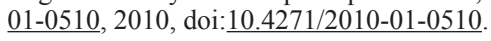

5. Duell, E. and George, A., "Experimental Study of a Ground Vehicle Body Unsteady Near Wake," SAE Technical Paper 1999-01-0812, 1999, doi: 10.4271/1999-01-0812.

6. Volpe, R., Devinant, P., \&Kourta, A. "Experimental characterization of the unsteady natural wake of the full-scale squareback Ahmed body: flow bi-stability and spectral analysis", Experiments in Fluids, 56:99, 2015.

7. Littlewood, R., \& Passmore, M., “Aerodynamic drag reduction of a simplified squareback vehicle using steady blowing", Experiments in Fluids, 53(2):519-529, 2012.

8. Littlewood, R., Passmore, M., and Wood, D., "An Investigation into the Wake Structure of Square Back Vehicles and the Effect of Structure Modification on Resultant Vehicle Forces," SAE Int. J. Engines 4(2):2629-2637, 2011, doi:10.4271/2011-37-0015.

9. Wood, D., Passmore, M., and Perry, A., "Experimental Data for the Validation of Numerical Methods - SAE Reference Notchback Model," SAE Int. J. Passeng. Cars - Mech. Syst. 7(1):145-154, 2014, doi:10.4271/2014-01-0590.

10. Wood, A., Passmore, M., Forbes, D., Wood, D. et al., "Base Pressure and Flow-Field Measurements on a Generic SUV Model," SAE Int. J. Passeng. Cars - Mech. Syst. 8(1):233-241, 2015, doi:10.4271/2015-01$\underline{1546 .}$.

11. Adrian, R., \& Westerweel, J. "Particle Image Velocimetry", Cambridge University Press, First Edition, 2011.

12. Elsinga, G. E., Scarano, F., Wieneke, B., \& van Oudheusden, B.W. "Tomographic Particle Image Velocimetry", Experiments in Fluids, 41:933-947, 2006.

13. Johl, G., Passmore, M., \& Render, P. "Design methodology and performance of an indraft wind tunnel", The Aeronautical Journal, 108:1087, 2004.

14. Howell, J., Sheppard, A., and Blakemore, A., "Aerodynamic Drag Reduction for a Simple Bluff Body Using Base Bleed," SAE Technical Paper 2003-01-0995, 2003, doi:10.4271/2003-01-0995.

15. Howell, J. and Le Good, G., "The Effect of Backlight Aspect Ratio on Vortex and Base Drag for a Simple Car-Like Shape," SAE Technical Paper 2008-01-0737, 2008, doi:10.4271/2008-01-0737.

16. Hollis, D. "Particle Image Velocimetry in Gas Turbine Combustor Flow Fields", Loughborough University Thesis, 2004.

17. Atkinson, C., \& Julio, S. “An Efficient Simultaneous Reconstruction Technique for Tomographic Particle Image Velocimetry", Experiments in Fluids, 47(4):553-568, 2009.

18. Wieneke, B. "Volume Self-Calibration for 3D Partcile Image Velocimetry", Experiments in Fluids, 45(4):549-556, 2008. 
19. Berkooz, G., Holmes, P., \& Lumley, L., "The Proper Orthogonal Decomposition in the Analysis of Turbulent Flows", Annual Review of Fluid Mechanics, 25:539-575, 1993.

20. Tropea, C., Yarin, A.L., \& Foss, J.F., "Springer Handbook of Experimental Fluid Mechanics: Part D, Chapter 22: Review of Some Fundamentals of Data Processing", Springer, 2007.

21. Pavia, G., Passmore, M., and Gaylard, A., "Influence of Short Rear End Tapers on the Unsteady Base Pressure of a Simplified Ground Vehicle. ," SAE Technical Paper 2016-01-1590, 2016, doi:10.4271/2016-01-1590.

\section{CONTACT INFORMATION}

A-K. Perry

01509227263

A.Perry@1boro.ac.uk

Almond

01509227263

M.Almond@lboro.ac.uk
Passmore

M.Passmore@lboro.ac.uk

Littlewood

rlittlewood@lavision.com

\section{ACKNOWLEDGMENTS}

The authors would like to thank David Cooper for the technical support he provided in preparation for the experiments and Dr Simon Tuplin for his assistance during testing. 


\section{APPENDIX}

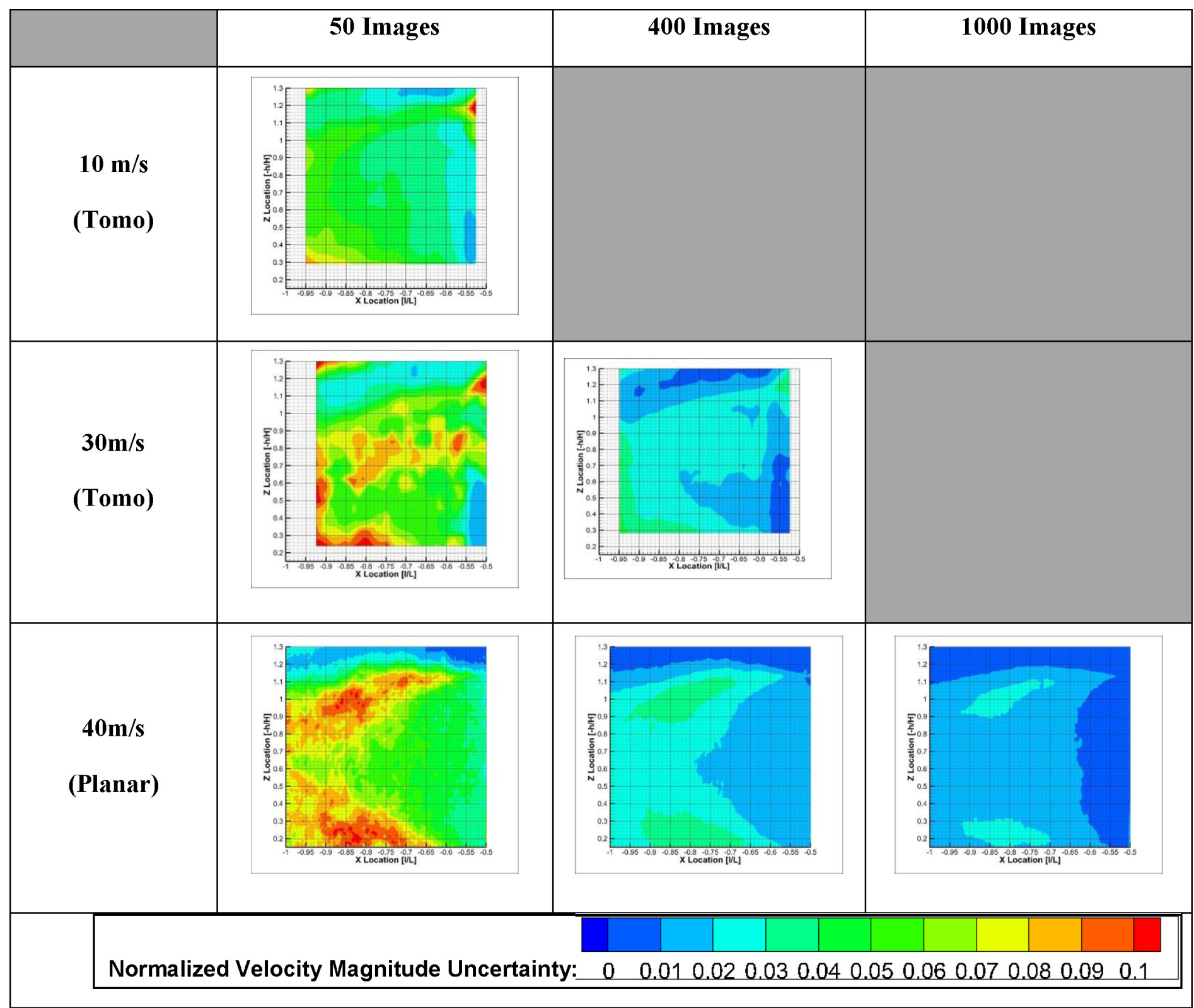

Figure 12. Uncertainty analysis showing the normalized $95 \%$ confidence interval of velocity magnitude in the time averaged velocity fields, constructed using varying data lengths. Results have been normalized using $\mathrm{V}_{\text {Tunnel }}$

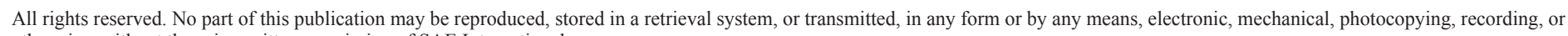
otherwise, without the prior written permission of SAE International. 